\title{
Innate immunity in COVID-19: Drivers of pathogenesis and potential therapeutic targets
}

\author{
Vichaya Ruenjaiman, ${ }^{1,2}$ Nattiya Hirankarn, ${ }^{1,2}$ Tanapat Palaga ${ }^{2,3}$
}

\begin{abstract}
A novel severe acute respiratory syndrome COVID-19 caused by coronavirus SARS-CoV-2 has been confirmed to infect more than 100 million people globally, with mortality reaching nearly 3 million as of March 2021. The symptoms vary widely, from the absence of any symptoms to death. The severity of COVID-19 relates to hyperinflammatory conditions with acute respiratory distress syndrome (ARDS), which leads to multiple-organ failure and death. Innate immunity plays an important role in the early response to SARS-CoV-2 infection and regulates the pathogenesis and its clinical outcomes. The most severe cases of COVID-19 present with increased innate immune cell infiltration in the lung, and elevated pro-inflammatory cytokines in the blood serum that are associated with disease severity. Here we review the innate immune response to SARS-CoV-2 infection based on the recent reports and discuss the potential roles of innate immune cells and their mediators in pathogenesis that dictate the outcome of the disease. Understanding the roles of innate immune responses at the initial stages of infection may provide early windows into treatment and clues for vaccine development.
\end{abstract}

Key words: COVID-19, SARS-CoV-2, Innate immunity, hyperimmune activation, immunopathology

From:

${ }^{1}$ Department of Microbiology, Faculty of Medicine, Chulalongkorn University, Bangkok, Thailand

${ }^{2}$ Center of Excellence in Immunology and Immune-Mediated Diseases, Chulalongkorn University, Bangkok, Thailand

Department of Microbiology, Faculty of Science,

Chulalongkorn University, Bangkok, Thailand

\section{Introduction}

The novel coronavirus SARS-CoV-2, which caused the outbreak of the severe acute respiratory syndrome, called COVID-19, was first identified and reported in Wuhan, China, in December 2019. In China, more than 90,000 cases of COVID-19 infection have been reported as of November 2020, and the infection has spread rapidly to other regions around the world. WHO declared COVID-19 a pandemic on 11 March 2020. ${ }^{1}$ As of March 2021, more than one hundred million people are confirmed to have been infected, with nearly three million deaths worldwide (refer to Johns Hopkins University Coronavirus Resource Center). In 2002 and 2011, the world had experienced severe respiratory syndromes caused by two other strains of coronavirus similar to COVID-19, i.e., severe acute respiratory syndrome (SARS) and Middle East respiratory syndrome (MERS). Whole genome sequencing of SARS-CoV-2 reveals $77.5 \%$ and $50 \%$ genome sequence identity to SARS-CoV and MERS$\mathrm{CoV}$, respectively. ${ }^{2}$ However, the infection of COVID-19 is more widespread and has a higher rate of mortality. The severity of COVID-19 has a wide spectrum of symptoms,

\section{Corresponding author:}

Tanapat Palaga

Department of Microbiology, Faculty of Science,

Chulalongkorn University, Bangkok, Thailand

E-mail: tanapat.p@chula.ac.th

with infected individuals being asymptomatic or having mild to severe symptoms. The factors that may influence symptoms include age, sex, underlying diseases, virus load, host genetics, and the host immune status. ${ }^{3}$

Most of the COVID-related deaths are due to acute respiratory distress syndrome (ARDS) and multiple-organ failure due to hyperimmune activation. Recent studies have highlighted the importance of innate immunity in controlling early response to SARS-CoV-2 infection and its detrimental roles upon dysregulation. Severe cases of COVID-19 have consistently shown increasing innate immune cell infiltration, especially neutrophils and inflammatory monocytes/macrophages in the lung and increasing pro-inflammatory cytokines in the blood serum, also known as cytokine storm. ${ }^{4}$ Here, we have focused on the innate immune responses to SARS-CoV-2, based on recent reports from patients as well as in vitro and in vivo studies in animal models. Combining insights from SARS and MERS, we propose a crucial function of innate immune response to SARS-CoV-2 and its detrimental effect, if not properly controlled in severe cases of COVID-19. 
Understanding how innate immunity to SARS-CoV-2 is dysregulated, which may exacerbate the disease, will provide early windows into treatment and clues for vaccine development.

\section{Recognition of SARS-CoV-2 by the host, and the interaction with innate immune cells Viral entry and replication}

The SARS-CoV-2 genome is a single-stranded RNA encoding a spike (S) glycoprotein, matrix (M), envelope (E), and nucleocapsid $(\mathrm{N})$ proteins, along with some accessory proteins. ${ }^{5}$ Angiotensin-converting enzyme 2 (ACE2) is known as the receptor for SARS-CoV-2 entry in human cells, which is a shared feature with SARS, but not MERS. ${ }^{6}$ ACE2 is a type I membrane protein expressed in type II alveolar cells. ${ }^{5} \mathrm{~A}$ heavily glycosylated $\mathrm{S}$ protein comprises of two subunits, S1 and S2. Virus entry depends on the binding of the receptor binding domain (RBD) of the S1 subunit to ACE2 on the host cells. ${ }^{6}$ After this, the $S$ protein is processed by the host cellular proteases, such as serine protease TMPRSS2 and furin, which allows protein cleavage at the S2 subunit. This event is followed by a conformational change, leading to membrane fusion and the release of viral genomic RNA into the cytoplasm. ${ }^{6}$ Consistent with this finding, serine protease inhibitor blocks SARS-CoV-2 infection of lung cells.

In addition to type II alveolar cells, ACE2 is also expressed in other cells, including esophageal epithelial cells, enterocytes of the small intestine, cholangiocytes, myocardial cells, proximal tubular cells of kidney, and bladder urothelial cells. ${ }^{7}$ This implies that SARS-CoV-2 could also infect other organs, resulting in non-respiratory symptoms in COVID-19 patients. An earlier study reported that tissue resident $\mathrm{CD} 169^{+}$macrophages express ACE2, implying that SARS-CoV-2 might directly infect macrophages as well. ${ }^{8}$ Moreover, SARS-CoV-2 can also infect $\mathrm{T}$ lymphocytes directly, resulting in lymphopenia in the patients. ${ }^{9}$ Antibody-dependent entry is yet another possibility, but no direct evidence is available.

\section{Antigen processing and presentation}

During viral replication, viral RNA is recognized as a pathogen-associated molecular pattern (PAMP) by patternrecognition receptors (PRRs). Currently, multiple molecules associated with SARS-CoV-2 are reported to be recognized as PAMPs. TLR3 and TLR7/8 are activated by endosomal dsRNA/ssRNA, and both RIG-I and MDA5 are activated by cytosolic dsRNA. The activation of TLR7/8 leads to the induction of transcription factors NF- $\mathrm{kB}$, and the expression of pro-inflammatory cytokines and chemokines (e.g., IL-6, TNF- $\alpha$, CXCL8 and CXCL10), whereas the activation of RIG-I and MDA5 leads to the induction of transcription factors IRF3 and IRF7, resulting in the expression of type I IFN (IFN- $\alpha$ and IFN- $\beta) .{ }^{10}$ Additionally, type I IFN binds to its receptor and activates the JAK/STAT signaling pathway via IRF9, resulting in the induction of IFN-stimulated genes (ISGs), such as RNASEL and CXCL1O (Figure 1). ${ }^{11}$ Genetic studies of familial genetic traits revealed that TLR3 and TLR7/8 may be the main TLRs for recognizing SARS-CoV-2 during the infection. ${ }^{12}$ For surface TLRs, TLR4 showed strongest binding interaction with the S protein of SARS-CoV-2 when compared to TLR1 and TLR6, suggesting that TLR4 signaling may be a mechanism driving immunopathology in COVID-19. ${ }^{13}$ Interestingly, aging human monocytes show impaired RIG-I signaling upon viral infection, and SARS-CoV-2 infection is most severe in aging patients. ${ }^{14}$ This observation suggests that RIG-I pathway may also recognize SARS-CoV-2 at the initial stages of infection.

Antigen presentation is a necessary immunological process to stimulate adaptive immunity. In coronavirus infection, both humoral and cellular immunity play an important role in mounting a successful antiviral response. Antigen presenting cells (APCs) at the entry sites, which are macrophages and dendritic cells, take up viral antigens, and process and present the antigens to $\mathrm{T}$ lymphocytes through major histocompatibility complex (MHC, or HLA in human)-T cell receptor (TCR) interaction. ${ }^{15} \mathrm{CD} 4^{+} \mathrm{T}$ cells or $\mathrm{T}$ helper cells recognize non-self antigens presented by the MHC class II on professional antigen-presenting cells, including dendritic cells, macrophages, some epithelial cells, and B cells. ${ }^{16}$ In contrast, $\mathrm{CD} 8^{+} \mathrm{T}$ cells recognize non-self antigens presented by MHC-I on nucleated cells, such as virus infected cells. The activation of $\mathrm{CD} 4^{+} \mathrm{T}$ cells leads to $\mathrm{B}$ cells maturation and generation of neutralizing antibody, which is necessary to eliminate the virus. ${ }^{16}$ Besides common APCs, pulmonary endothelial cells (ECs) express high levels of genes involved in MHC class II-mediated antigen presentation in lung tumors, implying that ECs may act as APCs as well. However, ECs do not express co-stimulatory molecules like the professional APCs, i.e., dendritic cells and macrophages..$^{17}$

An earlier study reported the depletion of tissue-resident alveolar macrophages, and a concomitant increase in inflammatory monocyte-derived macrophages in severe COVID-19 cases. $^{18}$ In SARS-CoV, which uses ACE2 for cellular entry similar to SARS-CoV-2, MHC class II is the main molecule presenting antigens to $\mathrm{T}$ cells. ${ }^{15}$ Moreover, previous studies reported the correlation between HLA polymorphisms and the susceptibility to SARS-CoV infection. ${ }^{19}$ However, the association between HLA gene polymorphisms and prognosis of patients in COVID-19 remains uncharacterized. This knowledge will help to predict the susceptibility of the patients to SARSCoV-2 infection, and maybe a target for therapeutic intervention, such as a vaccine.

\section{Innate immune response to SARS-CoV-2}

Innate immunity plays an essential role in controlling viral infection at an early stage to prevent viremia. Furthermore, the activation of innate immune cells is crucial for the initiation of adaptive immunity. Innate immune cells, such as macrophages, monocytes, dendritic cells, and neutrophils, detect viral infection using PRRs to recognize PAMPs and danger-associated molecular patterns (DAMPs). Hyperactivation of innate immune response is often associated with immunopathology and in coronavirus infections as in COVID-19 (Figure 2) and innate immune cells are essential for activating a specific and effective immune response to SARS-CoV-2 infection. 


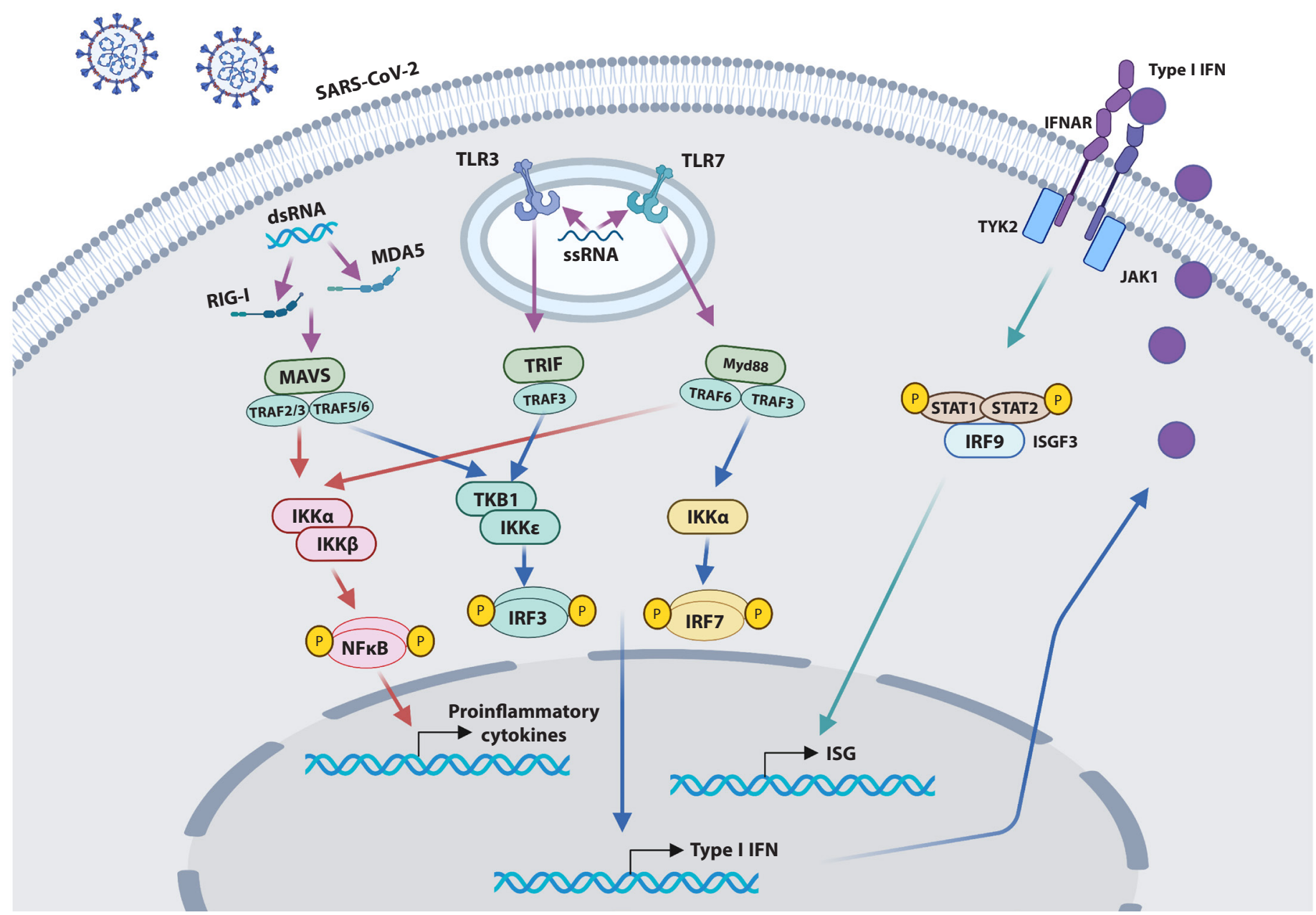

Figure 1. Innate immune response cascade to SARS-CoV-2 infection.

SARS-CoV-2 infection initial immune response by the activation of RNA recognition receptor including TLR3, TLR7/8, RIG-I and MDA5, resulting in the induction of downstream transcription factors NF- $\mathrm{B}$, IRF3 and IRF7 and the expression of pro-inflammatory cytokines, chemokines and type I IFN. Besides, type I IFN binds to its receptor and activates the JAK/STAT signaling pathway, resulting in the induction of IFN-stimulated genes (ISGs).

Figure created using Biorender (https://biorender.com/).

\begin{tabular}{|c|c|c|c|}
\hline Cell Stage & Normal activation & Hyper- or impair activation & SARS-CoV-2 infection \\
\hline Neutrophil & $\begin{array}{l}\text { - Inflammatory response } \\
\text { - Phagocytosis }\end{array}$ & $\begin{array}{l}\text { - Cytokine storm } \\
\text { - NET formation }\end{array}$ & $\begin{array}{l}\text { - Increase migration into the lung } \\
\text { - Production of proinflammatory cytokines } \\
\text { and chemokines } \\
\text { - NETs formation }\end{array}$ \\
\hline Monocyte and $M \varnothing$ & $\begin{array}{l}\text { - Inflammatory response } \\
\text { - Phagocytosis }\end{array}$ & $\begin{array}{l}\text { - Cytokine storm } \\
\text { - Increase cell proliferation of } \\
\text { inflammatory monocytes and } M \varnothing \\
\text { - Pyroptosis }\end{array}$ & $\begin{array}{l}\text { - Increase migration of } \mathrm{CCR} 2^{+} \text {monocytes into } \\
\text { the lung } \\
\text { - Production of proinflammatory cytokines } \\
\text { and chemokines }\end{array}$ \\
\hline & $\begin{array}{l}\text { - Antigen presentation } \\
\text { - Type I IFN secretion } \\
\text { - Promote NK, T cell function }\end{array}$ & $\begin{array}{l}\text { - Delay or impair IFN production } \\
\text { - Reduction of pathogen-specific T } \\
\text { cell response }\end{array}$ & $\begin{array}{l}\text { - Impair Type I IFN production } \\
\text { - Reduction of CD } 86 \text { and HLA-DR expression, } \\
\text { lead to decreasing of T cell response }\end{array}$ \\
\hline
\end{tabular}

Figure 2. Roles of innate immune cells in antiviral immunity at the normal stage, driven immunopathology at severe disease stages and COVID-19.

Figure created using Biorender (https://biorender.com/). 


\begin{tabular}{|c|c|c|c|}
\hline Cell Stage & Normal activation & Hyper- or impair activation & SARS-CoV-2 infection \\
\hline & $\begin{array}{l}\text { - Inflammatory response } \\
\text { - Tissue homeostasis }\end{array}$ & $\begin{array}{l}\text { - Promote inflammation by } \\
\text { degranulation, resulting in tissue } \\
\text { damage }\end{array}$ & $\begin{array}{l}\text { - Increase migration into the lung } \\
\text { - Associated with tissue damage, inducing } \\
\text { phagocytosis and cytokine production }\end{array}$ \\
\hline & $\begin{array}{l}\text { - Immuno suppressive } \\
\text { activity to prevent immune } \\
\text { overactivation }\end{array}$ & $\begin{array}{l}\text { - Massive expansion } \\
\text { - Suppress immune response, } \\
\text { especially T cell response }\end{array}$ & $\begin{array}{l}\text { - Massive expansion of MDSCs lead to reduc- } \\
\text { tion of immune response }\end{array}$ \\
\hline Innate & $\begin{array}{l}\text { - IFN- } \gamma \text { secretion } \\
\text { - Cytotoxic activity } \\
\text { - Antigen presentation } \\
\text { - Initiate adaptive immunity }\end{array}$ & $\begin{array}{l}\text { - Delay or impair IFN- } \gamma \text { production } \\
\text { - Decrease cytotoxicity }\end{array}$ & $\begin{array}{l}\text { - Reduction of circulate innate T cell } \\
\text { - Virus inhibit or delay IFN- } \gamma \text { production } \\
\text { - Increasing of activated CD } 69^{+} \text {innate T cells }\end{array}$ \\
\hline
\end{tabular}

Figure 2. (Continued)

\section{Involvement of neutrophils}

In viral infection, neutrophils are recruited at the site of infection early, and mediate the elimination of viruses using reactive oxygen species (ROS) and phagocytosis. Infected epithelial cells increase the production of neutrophil chemoattractants, resulting in neutrophils infiltration and accumulation in the infected tissue. ${ }^{20}$ The increase in neutrophils infiltration of the lung, and blood neutrophil-to-lymphocyte ratio are associated with disease severity and poor outcomes in COVID-19. ${ }^{4}$ Compared to other types of pneumonia, bronchoalveolar lavage fluid (BALF) from COVID-19 patients showed higher percentages of neutrophils and mast cells. ${ }^{21}$ Single-cell transcriptome analysis revealed that macrophages and neutrophils are the main innate immune cells present in the lung in severe COVID-19 cases. ${ }^{12}$ Higher pro-inflammatory cytokine and chemokine production in severe COVID-19 cases increases neutrophil activity related to immunopathology. One possible mechanism of neutrophil-driven immunopathology is neutrophil extracellular trap (NET) formation. NET is an extracellular web-like structure of chromatin and granular protein that is released extracellularly for killing pathogens. A massive NET formation can trigger inflammation and tissue damage in several diseases. ${ }^{22}$ Recent studies showed high levels of NET markers, including cell-free DNA, myeloperoxidase-DNA (MPO-DNA), and citrullinated histone $\mathrm{H} 3$ (Cit-H3), in the blood serum of COVID-19 patients, which may contribute to inflammation, microthrombosis, and ARDS in COVID-19. ${ }^{23}$ NET markers in blood serum from patients requiring mechanical ventilation are higher when compared to patients breathing naturally. Moreover, COVID-19 patient sera promote NET release from healthy control neutrophils in vitro. Currently, it is not known whether higher neutrophil activation is caused by virus per se or the effect of hyperimmune activation of other components.

\section{Critical roles of monocytes and macrophages}

Monocytes and tissue-resident macrophages are phagocytes that can be found in most tissues, and respond to infection as the first line of defense. ${ }^{24}$ In COVID-19, the activation of resident alveolar macrophages, $\mathrm{CD} 68^{+} \mathrm{CD} 169^{+}$macrophages of lymph nodes, and CCR2 ${ }^{+}$monocytes through PRRs triggers the production of type I IFN- $\gamma$ and other pro-inflammatory cytokines, such as IL-6, IL-1 $\beta$, and TNF- $\alpha$, that have effective antiviral functions, but also contribute to lung tissue damage and inflammation. ${ }^{25-27}$ Moreover, macrophages and monocytes produce high levels of chemokines, including CCL2, CXCL8, CXCL10, MCP1, and MIP1A, that can recruit other innate and adaptive immune cells to the site of infection. ${ }^{27}$ Consistent observations from various studies revealed that cytokines produced and secreted from macrophages, especially IL-6 and IL- $1 \beta$, are related to ARDS in COVID-19, and the increased macrophage and monocyte infiltration is the hallmark of tissue damage in severe COVID-19. ${ }^{27}$

Analysis at a single cell level in the lungs of COVID-19 patients revealed that differences between mild and severe COVID-19 cases can be attributed to overexpression of cytokines, including CCL2, CCL3, CCL20, IL8, IL1B, and TNF, in non-resident macrophages. This heightened response by inflammatory macrophages and epithelial cell interaction is the key component that drives tissue damage and respiratory failure. ${ }^{28}$ In a longitudinal study of COVID-19 patients, changes in phenotypes and functions in the $\mathrm{CD} 14^{+}$monocyte subset, including poor induction of COX2 and enhanced cycle marker Ki67, were reported. This result strongly indicated that monocytes/macrophages are the hallmark changes in COVID-19 patients. $^{29}$

\section{Roles of dendritic cells}

Dendritic cells (DCs) can be subdivided into at least two groups, viz., conventional DCs (cDCs) (also known as myeloid DCs [mDCs]), such as CD141 ${ }^{+} \mathrm{DC}(\mathrm{cDC} 1)$ and $\mathrm{CD} 1 \mathrm{c}^{+}$ DCs (cDC2), and the CD123 $3^{\text {hi }}$ plasmacytoid DCs (pDCs). $\mathrm{pDC}$ is a potent type I interferon secretor, which is crucial for antiviral mechanisms in several virus infections, such as HSV1, MCMV, influenza, and SARS-CoV, whereas $\mathrm{CDC}$ is a potent APC that can initiate an efficient antiviral adaptive immune response. ${ }^{30}$ DCs recognize viruses by TLR9 (for DNA viruses) and TLR7 (for RNA viruses), resulting in the secretion of type I interferons that have antiviral function and can contribute to immunopathology. ${ }^{31}$ In SARS-CoV infection, the mechanism 
of virus immune evasion is reported to be due to reduction or delay in the expression of type I antiviral cytokines, viz., IFN and IL-12p40, and increased expression of inflammatory chemokines in infected DCs. ${ }^{32}$ Besides increasing chemokine expression, DCs also increase the death receptor ligand TNF-related apoptosis-inducing ligand (TRAIL), which may be associated with lymphopenia in SARS patients. ${ }^{33}$ Moreover, cytokines IL-6 and IL-8 secreted from SARS-CoV infected epithelial cells can modulate the function of DCs by impairing their naïve $\mathrm{T}$ cell priming ability. ${ }^{34}$ COVID-19 patients showed a dramatic decrease in CD1c $\mathrm{c}^{+} \mathrm{DCs}$ in the blood during severe disease progression, which correlated with high inflammatory markers in blood serum and severe clinical outcomes. ${ }^{25}$ In severe COVID-19 cases, the frequency of pDCs in the periphery is reduced. Peripheral pDCs impair the production of IFN- $\alpha$ in response to TLR stimulation via the decrease in mTOR signaling. ${ }^{35}$ Furthermore, peripheral $\mathrm{mDC}$ s reduce the expression of CD86 and HLA-DR, which are crucial for antigen presentation and the induction of helper $\mathrm{T}$ cell response. ${ }^{35}$ This implies the association between impaired DC capacity in response to TLR stimulation and the reduction of virus-specific $\mathrm{T}$ cell response in severe COVID-19 cases.

\section{Decreasing blood granulocytes in severe COVID-19 cases}

Viruses can induce granulocyte degranulation, which results in both neutralizing viruses and contributing to immunopathology. ${ }^{36}$ The decrease in peripheral blood eosinophils and basophils has been reported in severe COVID-19 cases. Decreasing eosinophil count may indicate a poor outcome, and is related to higher viral load in COVID-19 patients. ${ }^{37}$ In contrast, the increase of eosinophils in blood after treatment may indicate a better outcome in COVID-19 patients. ${ }^{38}$ Another study found the rapid increase of eosinophil infiltration in the lungs of COVID-19 patients with pneumonia. ${ }^{39}$ In dengue virus infection, basophils are the target for antibody-enhanced virus infection, and play a role in the pathogenesis by the induction of vasoactive cytokines. ${ }^{40}$ For SARS-CoV-2 infection, the decrease of peripheral blood basophils may be because of the increase of basophil migration to the infection site. Moreover, CR3, which recognizes molecules associated with tissue homeostasis, especially fibrinogen, and plays a role in complement-mediated leukocyte recruitment, is significantly higher in granulocytes of COVID-19 patients requiring ventilatory support, and leads to increase in phagocytosis and cytokine production. ${ }^{41}$ This suggests that granulocytes play a crucial role in immunopathology of SARS-CoV-2 infection, and that CR3 may be used as a biomarker for tissue damage in severe COVID-19 cases.

\section{Myeloid-derived suppressor cells (MDSCs)}

MDSCs are a mixed population of myeloid cells that regulate immune response to prevent immune hyperactivation during infection, inflammation, and several diseases. Activation of MDSCs by proinflammatory mediators dampens the immune response to the tumor, resulting in promotion of tumor progression. Moreover, MDSCs have been reported to expand many pathological conditions. ${ }^{42}$ In COVID-19, massive expansion of MDSCs in peripheral blood mononuclear cells (PBMCs) of severe COVID-19 patients were observed compared with mild COVID-19 cases, implying that MDSCs respond to the inflammation driven by hyperactivation of the immune response and pro-inflammatory mediators. Thus, MDSCs may play an important role in limiting inflammation by immune suppressive function in COVID-19 patients. $^{43}$

Other innate immune cells: Mucosal-associated invariant $T$ (MAIT) cells, $\gamma \delta T$ cells, NKT cells, and innate lymphoid cells (ILCs)

Besides the classical innate immune cells as described above, there are other novel innate immune cells that may be associated with antiviral activity and immunopathology in SARS-CoV-2 infection, including invariant $\mathrm{T}$ cells and ILCs. Innate $\mathrm{T}$ cells comprise of MAIT, $\gamma \delta \mathrm{T}$ cells, and NKT cells, which contribute to the innate mucosal immunity, including the lung tissue. ${ }^{44}$ These groups of $\mathrm{T}$ cells recognize non-peptide antigens using non-classical MHC molecules, and are activated during the early stages of infection to initiate an adaptive immune response. COVID-19 patients show changes in the phenotypes and frequency of innate $\mathrm{T}$ cells in both blood and the airways. Most severe COVID-19 cases showed T cell lymphopenia, which has been correlated with disease severity. Frequency of circulating MAIT, NKT, and $\gamma \delta$ T cells was decreased in patients with severe COVID-19, whereas activated $\mathrm{CD}_{69^{+}} \mathrm{MAIT}, \mathrm{NKT}$, and $\gamma \delta \mathrm{T}$ cells was increased in the airways ${ }^{45,46}$ suggesting that the infiltration into the lung and the activation of innate $\mathrm{T}$ cells during SARS-CoV-2 infection may play a role in immunopathology. Another study on $\gamma \delta \mathrm{T}$ cell phenotypes in COVID-19 patients confirmed that these cells were dramatically decreased in the peripheral blood of patients. However, increase in $\mathrm{CD}^{+} \gamma \delta \mathrm{T}$ cells within the population was observed, implying that $\mathrm{CD}^{+} \gamma \delta \mathrm{T}$ cells may function in antigen presentation to initiate adaptive immunity during viral infection. ${ }^{47}$

ILCs are innate lymphocytes that can be dividing into five subsets, viz., natural killer (NK) cells, ILC1, ILC2, ILC3, and lymphoid tissue inducer (LTi) cells. ${ }^{48} \mathrm{NK}$ cells and ILC1 play a crucial role in antiviral infection by the secretion of IFN- $\gamma$, which activates $\mathrm{CD}^{+} \mathrm{T}$ cell antiviral activity. However, NK cells are different from ILC1 as they show cytotoxicity functions. ${ }^{48}$ In SARS-CoV-2 infection, total lymphocytes as well as NK cells are decreased in blood. ${ }^{49}$ ILC2 mainly produces $\mathrm{T}_{\mathrm{H}} 2$ cell-associated cytokines IL-5 and IL-13, whereas ILC3 and LTi cells mainly produce $\mathrm{T}_{\mathrm{H}} 17$ cell-associated cytokines IL-17 and IL-22 during the activation. ${ }^{50}$ COVID-19 patients showed a decrease in the number of circulating total ILC, and the alteration of ILC subsets. ${ }^{51}$ Moreover, ILC precursors (ILCp) decreased, whereas CD69+ ILCp and the percentage of ILC2, which plays a role in lung tissue repair, increased. ${ }^{51}$ IL-33 released by injured epithelial cells in the lung drives differentiation and activation of ILC2, which plays a role in pulmonary fibrosis. ${ }^{52}$ The result suggested that the infection may promote the maturation of circulating ILCp. Additionally, the increase of circulating ILC1 and reduction in circulating ILC2 were associated with severity in COVID-19. 


\section{Immunopathology related to innate immune re- sponse in COVID-19}

Most of the severely affected COVID-19 patients experience life-threatening complications, including ARDS, pneumonia, acute cardiac injury, acute kidney injury, shock, and multiple-organ failure. ${ }^{53}$ The immunopathology of severe COVID-19 stems from hyperactivation of the immune response, especially in the innate immunity, resulting in uncontrolled systemic inflammation (Figure 3).

\section{Cytokine storm}

Cytokine storm is the main mechanism of systemic inflammation-mediated immunopathology in severe COVID-19 cases. In most of the severe COVID-19 cases, infiltration of innate immune cells, such as neutrophils and macrophages, is increased in the lungs, leading to massive production of pro-inflammatory cytokines and chemokines, including IL-6, IL-1 $\beta$, IL-2, IL-8, IL-17, IL-10, G-CSF, GM-CSF, IFN- $\gamma$, IP10, MCP1, MIP1A, and TNF- $\alpha$. Furthermore, prominent acute inflammatory markers, such as acute phase reactants, including C-reactive protein, lactate dehydrogenase, and D-dimer, are also increased..$^{53}$ The release of a large number of pro-inflammatory cytokines, chemokines, and acute phase protein induces tissue damage, leading to ARDS, multiple-organ failure, and death associated with disease severity in COVID-19 patients.

\section{Pyroptosis}

Pyroptosis is a inflammasome-mediated and caspase 1-dependent program cell death in response to various pathological stimuli. The danger signals (PAMPS and DAMPS) trigger cell pyroptosis in macrophages and lymphocytes by the upregulation of inflammasome, especially NLRP3. This event leads to the activation of caspase 1 , which results in the processing of pro-inflammatory cytokines IL- $1 \beta$ and IL- $18 .{ }^{54}$ Previous studies reported the induction of pyroptosis in MERS$\mathrm{CoV}$ infection and the activation of NLRP3 inflammasome, which regulated IL-1 $\beta$ secretion in SARS-CoV infection. ${ }^{55,56}$ In SARS-CoV-2 infection, patients showed elevated serum IL-1 $\beta$, serum lactate dehydrogenase, neutrophil infiltration in the lung, and lymphopenia. ${ }^{37,53}$ Infiltration of lymphocytes and macrophages in the lung may cause an increase in cell death markers, which results in chronic pyroptosis activation. Therefore, pyroptosis activation may be one of the mechanisms promoting tissue damage and organ failure in SARSCoV-2 infection.

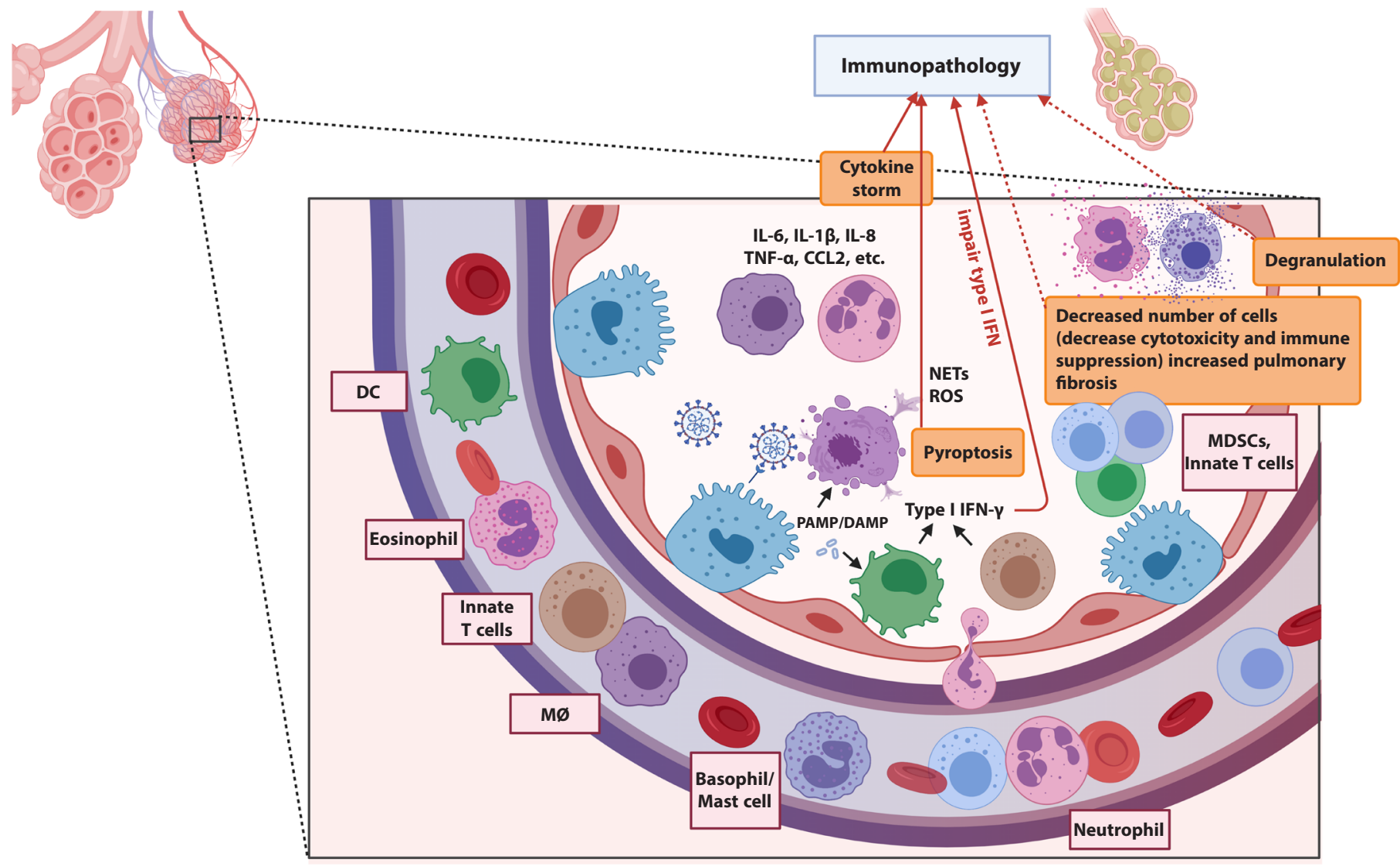

Figure 3. Schematic representation of virus-induced innate immune cell-driven immunopathology.

In COVID-19 patients, the virus induces an increase in the infiltration of innate immune cells, including neutrophils, macrophages, DCs, granulocytes, MDSCs, and other innate immune cells, in the lung, leading to cytokine storm, cell pyroptosis, NET formation, impaired Type I IFN production, degranulation of granulocytes, and various unknown effects that result in inflammation, lung tissue damage, and immunopathology.

Figure created using Biorender (https://biorender.com/). 


\section{Antiviral type I IFN}

Type I IFN is a subgroup of interferon that can be divided into many subtypes, including multiple IFN- $\alpha$, single IFN- $\beta$, IFN- $\varepsilon$, IFN- $\kappa$, and IFN- $\omega .{ }^{57}$ During viral infection, type I IFN is mainly produced by pDCs in response to TLR7 and TLR9 induction. ${ }^{57}$ Type I IFN functions by inducing anti-viral function, modulating innate immune response, and activating adaptive immune response. ${ }^{57} \mathrm{~A}$ previous study showed that delayed type I IFN response increases the accumulation of inflammatory monocytes and macrophages, resulting in immunopathology and promoting severe symptoms in SARS-CoV infection. ${ }^{58}$ Type I IFN also plays a role in driving the progression of COVID-19 severely. Classical monocytes in severe COVID-19 cases exhibit the upregulation of both, type I IFN and TNF/IL-1 $\beta$ responsive genes, which leads to hyper-inflammation-driven immunopathology. ${ }^{59}$ Longitudinal analyses showed delayed, but sustained high levels of type I IFN in severe COVID-19 cases, and the positive correlation between viral load with plasma IFNa, IFN $\gamma$, and TNF levels in moderate and severe cases. ${ }^{60}$ Moreover, other studies imply that impaired type I and III IFN was associated with persistent blood viral load and inflammatory responses, contributing to pathogenesis and mortality in COVID-19. ${ }^{58,61}$ Type I IFN drives the inflammation-mediated lung tissue damage by increasing influx of inflammatory innate immune cells, and inducing transcription factor $\mathrm{NFKB}$, leading to an increase in TNFa and IL-6. ${ }^{61}$ These results suggest that type I IFN is associate with inflammatory features in COVID-19 patients, and the impairment of type I IFN plays a critical role in immunopathology of severe COVID-19 cases.

The SARS-CoV-2 coronavirus is more transmissible than other coronaviruses and the resulted disease presents with wide spectrum of symptoms. Factors that dictate the clinical outcome identified to date include age, sex, underlying diseases, and immune status of the patient. ${ }^{3}$ The mechanisms of SARS-Cov- 2 immune evasion and immune response in SARSCov-2 infection have not been completely elucidated. However, many studies supported that innate immune modulation by virus plays a critical role in driving SARS-Cov-2 infectivity by delaying viral clearance and promoting immunopathology. One possible mechanism that may increase the transmission is the RBD of SARS-CoV-2 that exhibits higher affinity binding to its host receptor ACE2, compared to SARS-CoV. ${ }^{62}$ The dominant mutant D614G within the spike protein of SARSCoV-2 variants associate with increased viral entry and viral replication in the host cells and easier transmission. ${ }^{63}$ Moreover, SARS-CoV-2 evade the innate immune response and inhibits IFN signaling using several mechanisms including 1) impairing PRR recognition of the viral RNA and the structure of SARS-CoV-2 spike protein increase masking the RBD domain from neutralizing antibodies compared to SARS-CoV, ${ }^{62}$ leading to the increased viral replication and delayed innate immune response 2) IFN antagonists such as ORF3b are conserved in SARS-CoV-2 and more efficient in delaying IFN response than in SARS-CoV. ${ }^{64}$ Understanding how SARS-CoV-2 manipulate host innate immune response will not only help better understand the effect on host immune response but also facilitate a better vaccine strategy to target innate immune functions against this virus.

\section{COVID-19 immunotherapy targeting innate im- munity}

Presently, there are no vaccines or FDA-approved drugs specific to COVID-19 treatment. Ongoing clinical research is focusing on developing a new strategy for COVID-19 treatment. Current clinical treatments depend on the symptoms of patient, including anti-viral drugs, anti-inflammatory drugs, and supportive treatment. According to current studies, there are several therapeutic strategies targeting innate immunity that may be effective for COVID-19 treatment.

Several monoclonal antibodies against cytokine or cytokine receptor antagonists are being used to inhibit the inflammatory function of cytokines for preventing cytokine storm in COVID-19 patients. Tocilizumab treatment, which inhibits IL-6, effectively prevented ARDS in severe COVID-19 cases. ${ }^{65}$ Other studies reported that the treatment with recombinant IL-1RA (e.g., anakinra) and anti-GM-CSF (e.g., mavrilimumab) was associated with clinical improvement, especially respiratory function, in COVID-19 patients. ${ }^{66,67}$ Moreover, cytokine blocking antibodies, having anti-inflammatory functions, such as anti-IFN $\gamma$ (e.g., emapalumab) are also being tested in combination with anakinra (NCT04324021) on COVID-19 patients. Targeting other cytokines associated with the induction of pulmonary fibrosis, including IL-33 (e.g., astegolimab), IL-13 (e.g., lebrikizumab), IL-4 (e.g., dupilumab), IL-9 (e.g., MEDI-528), and IL-17 (e.g., bimekizumab) also are other options for fighting COVID-19, that may promote the resolution of inflammation. ${ }^{52}$ Besides cytokine-blocking antibodies, those targeting chemokines, such as CCL2 (e.g., carlumab), CCR5 (e.g., leronlimab), and CXCR2 (e.g., AZD5069), possibly inhibit tissue recruitment of inflammatory innate immune cells into the lungs, which might increase clinical improvement by decreasing lung inflammation. ${ }^{28}$ Although many studies have shown that cytokine storm is the main mechanism driving ARDS, some studies also showed that the activation of NLRP3 inflammasome, and the impairment of Type I IFN production could be the key to increase in disease severity in COVID-19 patients. Therefore, specific inhibitors, such as colchicine, dapansutrile, and CP-456773, for inhibiting the activation of NLRP3 inflammasome, and the administration of Type I IFN may be effective therapeutic strategies for the treatment of COVID-19. ${ }^{59,68}$

Besides the treatment using the conventional antiviral drugs or specific antibodies and inhibitors, there are many clinical studies on developing new treatment strategies that are more specific, safe, and effective for COVID-19. Most of these approaches aim to modulate the immune response to reduce the overactivation of the immune system and tissue repair. Mesenchymal stem cells (MSCs) are used for cell therapy in several immune-mediated inflammatory diseases. Intravenous administration of MSCs in severe COVID-19 cases resulted in an increase in the number of peripheral lymphocytes, regulatory DCs, and anti-inflammatory cytokine IL-10 levels, and a decrease in proinflammatory cytokine TNF- $\alpha$ level. ${ }^{69}$ Moreover, intravenous MSCs supported the repair of lung tissue, resulting in the reduction of lung pathology ${ }^{69}$ Presently, researchers have found a new type of macrophage named nerve and airway associated macrophages (NAMs), 
which has an immunoregulatory function. NAMs play a role in controlling the inflammation and tissue repair response to the virus in the lungs. ${ }^{70}$ Since NAMs function in immunoregulatory and lung tissue repair during viral infection, they may be used for cell therapy in COVID-19 like MSCs. Hence, the potential roles of innate immune cells and their mediators in pathogenesis, that dictate the outcome of the disease, may provide early windows into treatment and clues for vaccine development.

\section{Acknowledgments}

VR was supported by Postdoctoral Fellowship, Ratchadapisek Somphot Fund, Chulalongkorn University, Bangkok, Thailand. TP and NH were supported by National Research Council of Thailand (Project 811/2563) and the Program Management Unit for Human Resources \& Institutional Development, Research and Innovation - CU B16F630071.

\section{References}

1. Daga M, Kumar N, Aarthi J, Mawari G, Garg S, Rohatgi I. From SARS-CoV to Coronavirus Disease 2019 (COVID-19) -A Brief Review. J Adv Res. 2020;06:4-2019.

2. Kim J-M, Chung Y-S, Jo HJ, Lee N-J, Kim MS, Woo SH, et al. Identification of Coronavirus Isolated from a Patient in Korea with COVID-19. Osong Public Health Res Perspect. 2020;11(1):3-7.

3. Liu W, Tao Z-W, Wang L, Yuan M-L, Liu K, Zhou L, et al. Analysis of factors associated with disease outcomes in hospitalized patients with 2019 novel coronavirus disease. Chin Med J (Engl). 2020;133(9):1032-8.

4. Zhang B, Zhou X, Zhu C, Feng F, Qiu Y, Feng J, et al. Immune phenotyping based on neutrophil-to-lymphocyte ratio and IgG predicts disease severity and outcome for patients with COVID-19. Front Mol Biosci. 2020;7:157.

5. Cui J, Li F, Shi Z-L. Origin and evolution of pathogenic coronaviruses. Nat Rev Microbiol. 2019;17(3):181-92.

6. Hoffmann M, Kleine-Weber H, Schroeder S, Krüger N, Herrler T, Erichsen S, et al. SARS-CoV-2 Cell Entry Depends on ACE2 and TMPRSS2 and Is Blocked by a Clinically Proven Protease Inhibitor. Cell. 2020;181(2):271-80. e8.

7. Xin Zou KC, Jiawei Zou, Peiyi Han, Jie Hao, Zeguang Han. Single-cell RNA-seq data analysis on the receptor ACE2 expression reveals the potential risk of different human organs vulnerable to $2019-\mathrm{nCoV}$ infection. 2020;14(2):185-92.

8. Feng Z, Diao B, Wang R, Wang G, Wang C, Tan Y, et al. The Novel Severe Acute Respiratory Syndrome Coronavirus 2 (SARS-CoV-2) Directly Decimates Human Spleens and Lymph Nodes. medRxiv[Preprint]. 2020 [cited 2020Nov09]:Available from: https://www.medrxiv.org/content/10.1 101/2020.03.27.20045427v1

9. Wang X, Xu W, Hu G, Xia S, Sun Z, Liu Z, et al. SARS-CoV-2 infects T lymphocytes through its spike protein-mediated membrane fusion. Cell Mol Immunol. 2020;17(8):894.

10. Nelemans T, Kikkert M. Viral Innate Immune Evasion and the Pathogenesis of Emerging RNA Virus Infections. Viruses. 2019;11(10):961.

11. Lim YX, Ng YL, Tam JP, Liu DX. Human Coronaviruses: A Review of Virus-Host Interactions. Diseases. 2016;4(3):26.

12. Shaath H, Vishnubalaji R, Elkord E, Alajez NM. Single-Cell Transcriptome Analysis Highlights a Role for Neutrophils and Inflammatory Macrophages in the Pathogenesis of Severe COVID-19. Cells. 2020;9(11).

13. Choudhury A, Mukherjee S. In silico studies on the comparative characterization of the interactions of SARS-CoV-2 spike glycoprotein with ACE-2 receptor homologs and human TLRs. J Med Virol. 2020;92(10): 2105-13.

14. Molony RD, Nguyen JT, Kong Y, Montgomery RR, Shaw AC, Iwasaki A. Aging impairs both primary and secondary RIG-I signaling for interferon induction in human monocytes. Sci Signal. 2017;10(509):eaan2392.

15. Liu J, Wu P, Gao F, Qi J, Kawana-Tachikawa A, Xie J, et al. Novel Immunodominant Peptide Presentation Strategy: a Featured HLA-A* 2402-Restricted Cytotoxic T-Lymphocyte Epitope Stabilized by Intrachain Hydrogen Bonds from Severe Acute Respiratory Syndrome Coronavirus Nucleocapsid Protein. J Virol. 2010;84(22):11849.
16. Chiappelli F, Khakshooy A, Greenberg G. CoViD-19 Immunopathology and Immunotherapy. Bioinformation. 2020;16(3):219-22.

17. Goveia J, Rohlenova K, Taverna F, Treps L, Conradi L-C, Pircher A, et al. An Integrated Gene Expression Landscape Profiling Approach to Identify Lung Tumor Endothelial Cell Heterogeneity and Angiogenic Candidates. Cancer Cell. 2020;37(1):21-36.e13.

18. Liao M, Liu Y, Yuan J, Wen Y, Xu G, Zhao J, et al. The landscape of lung bronchoalveolar immune cells in COVID-19 revealed by single-cell RNA sequencing. medRxiv[Preprint]. 2020[cited 2020Nov09]:Available from: https://doi.org/10.1101/2020.02.23.20026690.

19. Wang S-F, Chen K-H, Chen M, Li W-Y, Chen Y-J, Tsao C, et al. Human-Leukocyte Antigen Class I Cw 1502 and Class II DR 0301 Genotypes Are Associated with Resistance to Severe Acute Respiratory Syndrome (SARS) Infection. Viral Immunol. 2011;24:421-6.

20. Wang S-Z, Forsyth KD. The interaction of neutrophils with respiratory epithelial cells in viral infection. Respirology. 2000;5(1):1-9.

21. Zhou Z, Ren L, Zhang L, Zhong J, Xiao Y, Jia Z, et al. Heightened Innate Immune Responses in the Respiratory Tract of COVID-19 Patients. Cell Host Microbe. 2020;27(6):883-90.e2.

22. Jorch SK, Kubes P. An emerging role for neutrophil extracellular traps in noninfectious disease. Nat Med. 2017;23(3):279-87.

23. Zuo Y, Yalavarthi S, Shi H, Gockman K, Zuo M, Madison JA, et al. Neutrophil extracellular traps in COVID-19. JCI Insight. 2020;5(11).

24. Martinez FO, Gordon S. The M1 and M2 paradigm of macrophage activation: time for reassessment. F1000Prime Rep. 2014;6:13.

25. Merad M, Martin JC. Pathological inflammation in patients with COVID-19: a key role for monocytes and macrophages. Nat Rev Immunol. 2020;20(6):355-62.

26. Wang $\mathrm{C}$, Xie J, Zhao L, Fei X, Zhang $\mathrm{H}$, Tan $\mathrm{Y}$, et al. Alveolar macrophage dysfunction and cytokine storm in the pathogenesis of two severe COVID-19 patients. EBioMedicine. 2020;57.

27. Huang C, Wang Y, Li X, Ren L, Zhao J, Hu Y, et al. Clinical features of patients infected with 2019 novel coronavirus in Wuhan, China. Lancet. 2020;395(10223):497-506.

28. Chua RL, Lukassen S, Trump S, Hennig BP, Wendisch D, Pott F, et al. COVID-19 severity correlates with airway epithelium-immune cell interactions identified by single-cell analysis. Nat Biotechnol. 2020;38(8): 970-9.

29. Mann ER, Menon M, Knight SB, Konkel JE, Jagger C, Shaw TN, et al. Longitudinal immune profiling reveals key myeloid signatures associated with COVID-19. Sci Immunol. 2020;5(51):eabd6197.

30. Pollara G, Kwan A, Newton PJ, Handley ME, Chain BM, Katz DR. Dendritic cells in viral pathogenesis: protective or defective? Int J Exp Pathol. 2005;86(4):187-204.

31. Jimenez-Baranda S, Greenbaum B, Manches O, Handler J, Rabadán R, Levine A, et al. Oligonucleotide Motifs That Disappear during the Evolution of Influenza Virus in Humans Increase Alpha Interferon Secretion by Plasmacytoid Dendritic Cells. J Virol. 2011;85(8):3893.

32. Law HKW, Cheung CY, Ng HY, Sia SF, Chan YO, Luk W, et al. Chemokine up-regulation in SARS-coronavirus-infected, monocyte-derived human dendritic cells. Blood. 2005;106(7):2366-74.

33. Law HKW, Cheung CY, Sia SF, Chan YO, Peiris JSM, Lau YL. Toll-like receptors, chemokine receptors and death receptor ligands responses in SARS coronavirus infected human monocyte derived dendritic cells. BMC Immunol. 2009;10:35.

34. Yoshikawa T, Hill T, Li K, Peters CJ, Tseng C-TK. Severe acute respiratory syndrome (SARS) coronavirus-induced lung epithelial cytokines exacerbate SARS pathogenesis by modulating intrinsic functions of monocyte-derived macrophages and dendritic cells. J Virol. 2009;83(7):3039-48.

35. Arunachalam PS, Wimmers F, Mok CKP, Perera RAPM, Scott M, Hagan $\mathrm{T}$, et al. Systems biological assessment of immunity to mild versus severe COVID-19 infection in humans. Science. 2020;369(6508):1210.

36. Walsh GM. Eosinophil granule proteins and their role in disease. Curr Opin Hematol. 2001;8(1).

37. Du Y, Tu L, Zhu P, Mu M, Wang R, Yang P, et al. Clinical Features of 85 Fatal Cases of COVID-19 from Wuhan: A Retrospective Observational Study. Am J Respir Crit Care Med. 2020;201(11):1372-9.

38. Liu F, Xu A, Zhang Y, Xuan W, Yan T, Pan K, et al. Patients of COVID-19 may benefit from sustained Lopinavir-combined regimen and the increase of Eosinophil may predict the outcome of COVID-19 progression. Int J Infect Dis. 2020;95:183-91. 
39. Kim D-M, Seo J-W, Kim Y, Park U, Ha N-Y, Park H, et al. Acute eosinophilic pneumonia associated with elevated NKT cell response in COVID-19 patients. Research Square[Preprint]; 2020[cited 2020Nov09]. p. Available from: https://doi.org/10.21203/rs.3.rs-3607/v1.

40. King CA, Marshall JS, Alshurafa H, Anderson R. Release of vasoactive cytokines by antibody-enhanced dengue virus infection of a human mast cell/basophil line. J Virol. 2000;74(15):7146-50.

41. Gupta R, Gant VA, Williams B, Enver T. Increased Complement Receptor -3 levels in monocytes and granulocytes distinguish COVID-19 patients with pneumonia from those with mild symptoms. Int J Infect Dis. 2020;99: 381-5.

42. Gabrilovich DI, Nagaraj S. Myeloid-derived suppressor cells as regulators of the immune system. Nat Rev Immunol. 2009;9(3):162-74.

43. Agrati C, Sacchi A, Bordoni V, Cimini E, Notari S, Grassi G, et al. Expansion of myeloid-derived suppressor cells in patients with severe coronavirus disease (COVID-19). Cell Death Differ. 2020.

44. Kawachi I, Maldonado J, Strader C, Gilfillan S. MR1-Restricted Va19<em $>\mathrm{i}</$ em $>$ Mucosal-Associated Invariant T Cells Are Innate T Cells in the Gut Lamina Propria That Provide a Rapid and Diverse Cytokine Response. J Immunol. 2006;176(3):1618-27.

45. Jouan Y, Guillon A, Gonzalez L, Perez Y, Ehrmann S, Ferreira M, et al. Functional alteration of innate $\mathrm{T}$ cells in critically ill Covid-19 patients. medRxiv[Preprint]. 2020[cited 2020Nov09]:Available from: http://medrxiv.org/content/early/2020/05/06/.05.03.20089300.abstract.

46. Jouan Y, Guillon A, Gonzalez L, Perez Y, Ehrmann S, Ferreira M, et al. Functional alteration of innate $\mathrm{T}$ cells in critically ill Covid-19 patients. medRxiv. 2020:2020.05.03.20089300.

47. Lei L, Qian H, Yang X, Zhou X, Zhang X, Zhang D, et al. The phenotypic changes of $\gamma \delta$ T cells in COVID-19 patients. J Cell Mol Med. 2020;24(19):11603-6.

48. Vivier E, Artis D, Colonna M, Diefenbach A, Di Santo JP, Eberl G, et al. Innate Lymphoid Cells: 10 Years On. Cell. 2018;174(5):1054-66.

49. Wang F, Nie J, Wang H, Zhao Q, Xiong Y, Deng L, et al. Characteristics of Peripheral Lymphocyte Subset Alteration in COVID-19 Pneumonia. J Infect Dis. 2020;221(11):1762-9.

50. Spits H, Artis D, Colonna M, Diefenbach A, Di Santo JP, Eberl G, et al. Innate lymphoid cells - a proposal for uniform nomenclature. Nat Rev Immunol. 2013;13(2):145-9.

51. García M, Kokkinou E, García AC, Parrot T, Palma Medina LM, Maleki KT, et al. Innate lymphoid cell composition associates with COVID-19 disease severity. Clin Transl Immunol. 2020;9:e1224.

52. Zizzo G, Cohen PL. Imperfect storm: is interleukin-33 the Achilles heel of COVID-19? Lancet Rheumatol. 2020;2(12):E779-90.

53. Wei L, Ming S, Zou B, Wu Y, Hong Z, Li Z, et al. Viral Invasion and Type I Interferon Response Characterize the Immunophenotypes during COVID-19 Infection. SSRN[Preprint]. 2020[cited 2020Nov09]:Available from: http://dx.doi.org/10.2139/ssrn.3564998.

54. Miao EA, Rajan JV, Aderem A. Caspase-1-induced pyroptotic cell death. Immunol Rev. 2011;243(1):206-14.
55. Chen IY, Moriyama M, Chang M-F, Ichinohe T. Severe Acute Respiratory Syndrome Coronavirus Viroporin 3a Activates the NLRP3 Inflammasome. Front Microbiol. 2019;10:50.

56. Jiang Y, Li J, Teng Y, Sun H, Tian G, He L, et al. Complement Receptor C5aR1 Inhibition Reduces Pyroptosis in hDPP4-Transgenic Mice Infected with MERS-CoV. Viruses. 2019;11(1):39.

57. Perry AK, Chen G, Zheng D, Tang H, Cheng G. The host type I interferon response to viral and bacterial infections. Cell Res. 2005;15(6):407-22.

58. Acharya D, Liu G, Gack MU. Dysregulation of type I interferon responses in COVID-19. Nat Rev Immunol. 2020;20(7):397-8.

59. Lee JS, Park S, Jeong HW, Ahn JY, Choi SJ, Lee H, et al Immunophenotyping of COVID-19 and influenza highlights the role of type I interferons in development of severe COVID-19. Sci Immunol. 2020;5(49):eabd1554.

60. Lucas C, Wong P, Klein J, Castro TBR, Silva J, Sundaram M, et al. Longitudinal analyses reveal immunological misfiring in severe COVID-19. Nature. 2020;584(7821):463-9.

61. Hadjadj J, Yatim N, Barnabei L, Corneau A, Boussier J, Smith N, et al. Impaired type I interferon activity and inflammatory responses in severe COVID-19 patients. Science. 2020;369(6504):718.

62. Wrapp D, Wang N, Corbett KS, Goldsmith JA, Hsieh C-L, Abiona O, et al. Cryo-EM structure of the 2019-nCoV spike in the prefusion conformation. Science. 2020;367(6483):1260.

63. Plante JA, Liu Y, Liu J, Xia H, Johnson BA, Lokugamage KG, et al. Spike mutation D614G alters SARS-CoV-2 fitness. Nature. 2020.

64. Konno Y, Kimura I, Uriu K, Fukushi M, Irie T, Koyanagi Y, et al. SARS-CoV-2 ORF3b Is a Potent Interferon Antagonist Whose Activity Is Increased by a Naturally Occurring Elongation Variant. Cell Rep. 2020;32(12):108185.

65. Qin C, Zhou L, Hu Z, Zhang S, Yang S, Tao Y, et al. Dysregulation of immune response in patients with COVID-19 in Wuhan, China. Clin Infect Dis. 2020;71(15):762-8.

66. Cavalli G, De Luca G, Campochiaro C, Della-Torre E, Ripa M, Canett $\mathrm{D}$, et al. Interleukin-1 blockade with high-dose anakinra in patients with COVID-19, acute respiratory distress syndrome, and hyperinflammation: a retrospective cohort study. Lancet Rheumatol. 2020;2(6):E325-31.

67. De Luca G, Cavalli G, Campochiaro C, Della-Torre E, Angelillo P, Tomelleri A, et al. GM-CSF blockade with mavrilimumab in severe COVID-19 pneumonia and systemic hyperinflammation: a single-centre, prospective cohort study. Lancet Rheumatol. 2020;2(8):e465-e73.

68. Shah A. Novel Coronavirus-Induced NLRP3 Inflammasome Activation A Potential Drug Target in the Treatment of COVID-19. Front Immunol. 2020;11:1021.

69. Leng Z, Zhu R, Hou W, Feng Y, Yang Y, Han Q, et al. Transplantation of ACE2(-) Mesenchymal Stem Cells Improves the Outcome of Patients with COVID-19 Pneumonia. Aging Dis. 2020;11(2):216-28.

70. Ural BB, Yeung ST, Damani-Yokota P, Devlin JC, de Vries M, Vera-Licona $\mathrm{P}$, et al. Identification of a nerve-associated, lung-resident interstitial macrophage subset with distinct localization and immunoregulatory properties. Sci Immunol. 2020;5(45):eaax8756. 